\title{
LA HERMENEUTIZACIÓN DE LA FILOSOFÍA DE LA CIENCIA CONTEMPORÁNEA*
}

AMBROSIO VELASCO GÓMEZ

INSTITUTO DE INVESTIGACIONES FILOSÓFICAS

UNIVERSIDAD NACIONAL AUTÓNOMA DE MÉXICO

\section{Introducción}

Desde el siglo pasado, en la filosofía de la ciencia social han predominado dos tradiciones opuestas: la empirista, que incluye la positivista, y la tradición hermenéutica. La primera se ha desarrollado básicamente en la filosofía de la ciencia anglosajona y la segunda en la filosofía, historiografía y sociología continental, principalmente alemana. Cada una de ellas caracteriza el lenguaje, estructura, objetivos, metodologías, criterios de validez y desarrollo de las ciencias socio-históricas de manera distinta. La separación entre estas concepciones refleja la distinción aristotélica entre las ciencias teóricas que son demostrativas y el saber práctico que no lo es. Salvo esporádicos debates, estas dos tradiciones se han mantenido distantes con mutua indiferencia, asumiendo cada una que se trata de teorías filosóficas incompatibles, entre las cuales el diálogo crítico es casi imposible y poco fructifero.

La tradición empirista se ha preocupado fundamentalmente por analizar y elucidar los argumentos que permiten demostrar la verdad o falsedad de los enunciados científicos, y con ello, distinguir claramente entre ciencia y metafísica. En la búsqueda de este criterio metodológico de cientificidad o criterio de demarcación, los filossofos de esta tradición empirista han entablado fuertes discusiones sin encontrar una solución que gane consenso. ${ }^{1} \mathrm{Ni}$ el criterio de verificabilidad empírica de los positivistas, ni el de falsabilidad o refutabilidad empírica de Popper, ni tampoco los criterios de confirmabili-

* Agradezco los comentarios del doctor Carlos López Beltrán a una versión anterior presentada en el Simposio XIV Internacional de Filosofía, organizado por el Instituto de Investigaciones Filosoficas.

1 Cfr. C. Hempel, "Problemas y cambios del criterio empirista de significado" en A.J. Ayer, El positivismo lógico, Fondo de Cultura Económica, 1981. 
dad, desarrollados por autores como Hempel, pudieron dar respuesta cabal a importantes objeciones lógicas, semánticas e históricas.

A través de estos debates dentro dę la tradición anglosajona, las concepciones sobre el proceder de los científicos para aceptar o rechazar las teorías e hipótesis de una manera racionalmente justificada han cambiado significativamente, dando origen a lo que se conoce como la concepción "pospositivista", "poskuhniana" o como prefiero nombrarla "pospopperiana". Básicamente, esta concepción filosófica de la ciencia se opone a concepciones positivistas de la ciencia en los siguientes puntos:

a) No existe una clara separación entre términos teóricos y términos observacionales, sino que todo término es teóricamente dependiente. Consecuentemente,

b) no existe un lenguaje objeto, observacional o fisicalista incuestionable, como lo proponían los positivistas (Carnap), con base en el cual se definan todos los predicados de las ciencias fácticas y se construyan los enunciados protocolarios que sirvan de base empírica natural para la contrastación (verificación o refutación) de hipótesis y teorías. Consecuentemente,

c) la base empírica de toda teoría, además de estar "preñada" de contenidos y presupuestos teóricos, es resultado de complejos procesos de comunicación y establecimiento de consensos entre los científicos. Tales procesos no son susceptibles de reconstrucción exhaustiva en términos de reglas o procedimientos metodológicos. Consecuentemente,

d) las teorias científicas están empíricamente subdeterminadas, tanto en un sentido semántico (reducción al lenguaje observacional) como en un sentido metodológico (no existe un conjunto de reglas claras que permita decidir de manera concluyente la verdad o falsedad de un enunciado científico).

Esta concepción de la ciencia que se ha desarrollado a partir de Popper constituye un serio cuestionamiento a la racionalidad del conocimiento científico. Paradójicamente, los filósofos de la ciencia contemporáneos (Popper, Kuhn, Lakatos, Shapere, Hesse, Laudan, etc.), en su empeño por explicar de manera objetiva el carácter distintivo de la racionalidad científica, han descubierto que bajo los criterios, presupuestos y métodos de análisis sustentados por los positivistas lógicos, la ciencia parece no ser una empresa tan racional como ellos pensaban. Lejos de abandonar la fe en la racionalidad científica, los filósofos pospopperianos de la ciencia se han preocupado por elaborar conceptos alternativos de la racionalidad de la 
ciencia que desbordan los límites establecidos por los positivistas e inclusive por el mismo Popper. ${ }^{2}$

La tesis principal de este trabajo es que en estos nuevos intentos por explicar la racionalidad científica, los más destacados representantes de la filosofía de la ciencia anglosajona han virado hacia un acercamiento o convergencia con tesis centrales de una tradición alternativa: la hermenéutica.

Esta hermeneutización de la filosofía de la ciencia pospositivista se debe no tanto al diálogo con los filósofos de la tradición hermenéutica, sino más bien al influjo decisivo que a partir de Popper ha tenido un filósofo prepositivista: Pierre Duhem. En el siguiente inciso se expondrán algunas de las ideas de Duhem que han influido a autores como Popper, Lakatos y Laudan, entre otros, en sus concepciones no positivistas. Posteriormente, trataré de mostrar las convergencias y analogías de estas concepciones con tesis centrales de filósofos hermeneutas. Finalmente, a manera de conclusión se discutirán algunos de los problemas centrales que se derivan de esta convergencia y que pueden constituir preguntas interesantes para una filosofía de la ciencia hermenéuticamente orientada. ${ }^{3}$

\section{Duhem ${ }^{4}$ y la filosofía de la ciencia pospopperiana}

A principios del siglo XX, Pierre Duhem, en su libro The Aim and Structure of Physical Theory, defendió una idea de las teorías físicas que resultó incompatible con las concepciones positivistas. Duhem consideraba, con cierto tono positivista, que "el acuerdo con el experimento es el único criterio de verdad para una teoría física". ${ }^{5}$ Pero Duhem sostenía que "un experimento en física es la observación precisa de los fenómenos acompañados por una interpretación de esos fenómenos; tal interpretación sustituye los datos concretos realmente recolectados a través de la observación por una correspondiente representación abstracta y simbólica, con base en teorías

2 "Si la historia o cualquier otra disciplina empírica nos lleva a creer que el desarrollo de la ciencia depende de un comportamiento que previamente hemos considerado irracional, entonces deberíamos concluir no que la ciencia es irracional, sino que nuestra noción de racionalidad necesita retoques aquí y allá", T.S. Kuhn, "Notas sobre Lakatos" en I. Lakatos y Musgrave (eds.), La crítica y el desarrollo del conocimiento, Grijalbo, Barcelona, 1975, p. 520.

3 Ulises Moulines ha reconocido que la filosofía de la ciencia es una disciplina hermenéutica. Véase U. Moulines, Pluralidad y recursión, Alianza Universidad, Madrid, 1991. Pero este reconocimiento se refiere a la filosofía de la ciencia como una metaciencia, no a la ciencia misma. Aquí trataremos de demostrar que las ciencias mismas, vistas desde la filosofía contemporánea (pospopperiana) tienen un carácter hermenéutico.

4 Agradezco a Larry Laudan sus observaciones respecto a la gran influencia de las ideas de Pierre Duhem en la filosofía pospositivista de la ciencia.

5 Pierre Duhem, The Aim and Structure of Physical Theory, Atheneum, Nueva York, 1977, cap. II, sec. 1, p. 21. [La traducción de los textos citados es mía.] 
previamente aceptadas por el observador". ${ }^{6}$ Esta tesis sobre la dependencia teórica de la observación y la experimentación en la ciencia se opone radicalmente al principio fundamental del positivismo lógico sobre la existencia de un lenguaje observacional teóricamente neutro, con base en el. cual se definan con precisión todos los términos no lógicos de las teorías científicas y gracias a ello sea posible verificar hipótesis científicas.

La visión antipositivista que Duhem desarrolló con antelación al auge del positivismo lógico ha generado en la filosofía de la ciencia pospopperiana un cuestionamiento profundo a la concepción positivista de la racionalidad cientifica. En especial, una consecuencia importante de la tesis de Duhem desarrollada por Karl R. Popper es la idea de que "la base empírica" de toda teoría científica tiene que aceptarse por acuerdo o convención, ya que esa base empírica, al estar "preñada" de teoría, no puede estar fundamentada en la observación pura, como pretendían los positivistas. ${ }^{7}$ A su vez, el reconocimiento de la dependencia teórica de la base empírica conduce también a admitir que las teorías científicas están "empíricamente subdeterminadas" y que, por lo tanto, la verificación empírica de hipótesis y leyes no está justificada.

La crisis de la concepción positivista de la ciencia a partir del problema planteado por Duhem y desarrollado por Popper y otros filósofos contemporáneos de la ciencia, se agudizó aún más con los trabajos de Thomas $\mathrm{S}$. Kuhn a principios de los años 60 . Kuhn radicalizó el convencionalismo (ya aceptado por Popper en forma limitada), al defender la idea de que no sólo la base empírica, sino también los principios e hipótesis fundamentales de toda teoría son aceptados por convención entre los científicos, a pesar de que exista evidencia empírica en su contra. En última instancia, la aceptación o rechazo de las teorías científicas no depende en lo fundamental de la evidencia empírica disponible, sino de los compromisos de la comunidad de científicos con ciertos presupuestos metafísicos y metodológicos. En este sentido, Kuhn no sólo acepta la tesis de la subdeterminación empírica de las teorías en un sentido positivo (esto es, la imposibilidad de verificar empíricamente hipótesis o leyes generales), sino también en un sentido negativo (la imposibilidad de refutar con base en mera evidencia empírica una teoría bien atrincherada en el paradigma o tradición a la que pertenece).

En todo caso, la contrastación de una hipótesis es un problema de competencia entre teorías. En caso de contradicción entre la teoría interpretativa de la observación y las hipótesis teóricas explicativas, no hay algoritmo ni regla metodológica alguna que permita decidir de manera justificada cuál

6 Ibidem., cap. IV, sec. 1, p. 147.

7 Cfr. Karl R. Popper, La lógica de la investigación científica, Tecnos, Madrid, 1973, cap. 5, "El problema de la base empirica". 
de ellas es la correcta. La decisión estriba en los compromisos del científico con una u otra teoría. Lakatos y poseriormente Laudan tratarán de proponer algunos criterios para salvar las consecuencias relativistas de la opinión radical de Kuhn sobre la subdeterminación empírica de las teorías, y reconstruir la tambaleante racionalidad científica. De nuevo, en este intento, estos filósofos recurrirán a Pierre Duhem. ${ }^{8}$

Lakatos medió y sacó provecho de la polémica entre Popper y Kuhn sobre la racionalidad del cambio y la permanencia de teorías. Su concepto de "programas de investigación" reconoce y concilia la tenacidad de las teorias científicas (tesis kuhniana) con su refutación y cambio (tesis popperiana). Según Lakatos, el núcleo teórico básico de un programa de investigación es irrefutable por acuerdo entre los científicos que trabajan en ese programa. En caso de que algunas tesis de este núcleo básico resulten amenazadas por alguna evidencia empírica, lo que se cambia no es la tesis cuestionada, sino hipótesis auxiliares que constituyen un cinturón de protección del núcleo básico. El criterio último de racionalidad para preservar el núcleo básico y con ello mantener el programa de investigación correspondiente estriba en su capacidad para producir eventualmente predicciones exitosas (progreso empírico). Qué tan eventuales pueden ser esas predicciones es algo que depende del "buen sentido" metodológicamente adiestrado de los científicos. Siguiendo (y complementando) a Pierre Duhem, Lakatos considera que la racionalidad de la decisión de hasta cuándo mantener un programa de investigación y cuándo abandonarlo por otro, no se puede expresar o sintetizar en una simple regla o "Ley estatutaria", como los positivistas y Popper pretendían, sino más bien se expresa a través de una experiencia, de

8 Es interesante la solución que Duhem da a una situación de conflicto entre teorfa interpretativa y teoría explicativa al comparar la estrategia seguida por un físico audaz que decide cambiar las hipótesis explicativas fundamentales (estrategia que defiende Popper), con un científico conservador que prefiere modificar algunas hipotesis auxiliares para salvar la teoría explicativa (estrategia típica de la ciencia normal, según Kuhn): "ya que la lógica no determina con precisión estricta cuándo una hipótesis inadecuada debe dar paso a otra más fructífera y dado que el reconocimiento del momento adecuado para tal cambio depende del buen sentido, los físicos deben ponderar sus juicios e incrementar la rapidez del progreso científico tratando de desarrollar de manera consciente dentro de ellos el buen sentido, de manera más lúcida y vigilante. Ahora bien, las pasiones y los intereses son los que más contribuyen a obstaculizar el buen sentido y perturbar su visión. Por ello, nada dilatará más la decisión que determinará una reforma afortunada de la teoría física que la vanidad que hace a un físico ser demasidado indulgente con su propio sistema y demasido severo con el sistema de otro". (P. Duhem, op. cit., cap. VI, sec. 10, p. 218.) [Las cursivas son mías.]

9 "En lugar de dejar que el inarticulado sentido común de Duhem juzgue cuándo debe ser abandonado un marco general, yo inyecto algunos solidos elementos popperianos en la evaluación de si un programa progresa o degenera." I. Lakatos, "La historia de la ciencia y sus reconstrucciones racionales" en su op. cit., p. 466. 
una sabiduría, producto de la "jurisprudencia" ${ }^{10}$ que los científicos desarrollan a través de la comunicación y el establecimiento de acuerdos. El valor fundamental que orienta esta sabiduría es el progreso científico, entendido como incremento de los éxitos predictivos y explicativos del programa de investigación en cuestión.

Larry Laudan ha elaborado con mayor precisión la idea de progreso científico. Al igual que Kuhn y Lakatos, Laudan ubica las teorías científicas dentro de unidades más complejas a las que denomina "tradiciones". Las tradiciones de investigación están constituidas en lo fundamental por un conjunto de presupuestos metafísicos y metodológicos que orientan la elaboración, aceptación y cambio de teorías dentro de la tradición en cuestión. ${ }^{11} \mathrm{Al}$ igual que Kuhn, pero más a tono con la concepción de Lakatos, Laudan se preocupa por elucidar criterios racionales que los científicos utilizan para evaluar las tradiciones con las que están comprometidos. Laudan también considera que la racionalidad de las tradiciones está en función del progreso científico, pero, a diferencia de Lakatos, distingue diferentes contextos y criterios para evaluar el progreso de las tradiciones. Por una parte, Laudan considera el contexto de "aceptación" y su correspondiente noción de "progreso global", y por otra parte, el "contexto de prosecusión" o "búsqueda", al que corresponde el criterio de "tasa de progreso". El contexto de aceptación se refiere al incremento acumulativo total de éxitos (predictivos y explicativos) de una tradición en un periodo dado, mientras que la tasa de progreso se refiere a la rapidez del incremento de explicaciones y predicciones. Así pues, ambos contextos y criterios de racionalidad de las tradiciones científicas se basan en el cúmulo de evidencia empírica que la sustenta, pero mientras que en el contexto de aceptación la evidencia cuenta como fundamento de corroboración empírica de las teorías, en el contexto de la prosecución la evidencia constituye la garantía del potencial heurístico de la tradición de investigación en cuestión.

En suma, las teorías filosóficas de las ciencias naturales de Popper, Lakatos y Laudan, entre otros destacados filósofos contemporáneos de la tradición anglosajona, además de retomar la tesis antipositivista de Duhem respecto a la dependencia teórica de toda observación, también retoman la idea básica del filósofo y físico francés de buscar los criterios de aceptación o rechazo de teorías en una sabiduría práctica, jurisprudencia o buen sentido que los científicos desarrollan en su trabajo cotidiano dentro de programas

10 "la sabiduría del jurado científico y su jurisprudencia no han sido, ni pueden ser, completamente articuladas por la ley estatutaria del filósofo. .. ", Lakatos, ibidem., p. 490.

11 Cfr. L. Laudan, Progress and its Problems, California University Press, 1977, cap. III y L. Laudan, "Un enfoque de solución de problemas al progreso cientifico" en I. Hacking (comp.), Las revoluciones científicas, Fondo de Cultura Económica, México, 1985. 
de investigación o tradiciones cientificas. Esta capacidad corresponde más al campo de juicio prudente que al de la demostración lógica.

Es importante señalar que la actitud que ha conducido a los filosofos contemporáneos a reconocer este campo más amplio y plural de la racionalidad cientifica es doble: por una parte una actitud descriptivista (naturalista) respecto a las ciencias y su historia, que les ha obligado a abandonar las nociones apriorísticas y restringidas de racionalidad, tal y como los positivistas (e inclusive Popper) la concebían; por otro lado, un rechazo al relativismo epistemologico, acompañado de una tenaz preocupación por reconocer nuevas y más auténticas formas de racionalidad científica. En este doble trabajo de crítica y reconstrucción, filósofos de la tradición anglosajona a las que nos hemos referido y otros como Toulmin, Hesse, Shapere, Hacking, entre otros, han arribado a tesis incompatibles con el positivismo lógico y coincidentes con principios fundamentales de la tradición hermenéutica. Estas tesis de convergencia pueden sintetizarse en los siguientes puntos:

1. Toda investigación científica parte de presupuestos conceptuales, teóricos, metodológicos y axiológicos, no siempre explícitamente formulables ni mucho menos cuestionables, que guían de una manera determinante la observación, la formulación y contrastación de hipótesis y teorías, así como las decisiones respecto a la aceptación, rechazo o modificación de las teorías en cuestión.

2. La validez de una teoría científica, su aceptación o rechazo no pueden determinarse en función del grado de corroboración de su hipótesis y leyes fundamentales sobre una base empírica segura y firme. Dicha evaluación tiene que hacerse en términos de su contribución al desarrollo de cuerpos de conocimientos más complejos como paradigmas, programas o tradiciones de investigación. Sólo dentro del movimiento de estas unidades más complejas y abarcadoras es posible apreciar la racionalidad científica. ${ }^{12}$

3. La racionalidad de las ciencias está determinada por las consecuencias globales de la resolución de las tensiones que constantemente emergen entre los presupuestos y convenciones de una tradición y las propuestas innovadoras que los cientficos elaboran en su trabajo dentro de la tradición. En la medida en que estas consecuencias contribuyan a un cambio progresivo de la tradición, tanto en rérminos de adecuación empírica como de capacidad heurística, la actividad científica será una empresa racional.

12 De aqui se desprende que la comprensión y reconstrucción de la racionalidad cientfica exige una interpretación histórica del desarrollo de la teoría dentro de su correspondiente tradición. 
4. La resolución de las tensiones dentro de una tradición entre lo aceptado previamente y la innovación, entre las hipótesis explicativas y la interpretación de lo observado, no puede realizarse por medio de algún procedimiento metodológico, preciso e invariante. Las tensiones esenciales no pueden resolverse por el simple recurso a un determinado algoritmo metodológico. La solución exitosa de estas tensiones depende de un juicio prudente de la comunidad científica competente a través de procesos comunicativos, argumentales, persuasivos y consensuales que desbordan con mucho cualquier criterio metodológico de demarcación.

A continuación, trataré de mostrar el carácter hermenéutico de estas cuatro tesis de la filosofía de la ciencia pospopperiana, a partir de un breve análisis de algunas ideas de Hans Georg Gadamer.

\section{Gadamer y la tradición hermenéutica}

Desde sus inicios con W. Dilthey, la hermenéutica contemporánea se ha propuesto elaborar una teoría filosófica de la ciencia social, como alternativa contestataria a la pretensión positivista de imponer su concepción de las ciencias naturales sobre las "ciencias de la cultura" o "ciencias del espíritu". ${ }^{13}$ Desde la perspectiva hermenéutica, las ciencias de la culțura buscan comprender e interpretar los textos y acciones humanas, más que explicar y predecir hechos, como ocurriría en las ciencias naturales.

Dentro de la tradición hermenéutica, un principio fundamental que se ha ido radicalizando es el reconocimiento de que toda comprensión parte de conceptos, creencias y concepciones establecidas de antemano. Aun autores como Dilthey, que se preocupan por rescatar un significado original de las acciones, reconocen la dependencia del intérprete y del agente respecto a un determinado conjunto de valores, creencias y hábitos propios del contexto. ${ }^{14}$

13 Sobre el desarrollo histórico de la hermenéutica contemporánea y su polémica con el positivismo, véase mi artículo "Filosofía de la ciencia, hermenéutica y ciencias sociales", Ciencia y desarrollo, vol. XXI, no. 125, nov.-dic., 1995.

14 Dilthey afirma que la "mente objetiva" (objective mind) es el contexto de creencias a partir del cual se realiza toda comprensión. Por mente objetiva entiende "las múltiples formas en las que lo que es común a los individuos se objetiviza en el mundo de los sentidos. En esta mente objetiva el pasado persiste permanentemente en nuestro presente. Su ámbito se extiende de los estilos de vida y formas de interacción social a los sistemas de propósitos que la sociedad ha creado y a las costumbres, ley, estado, religión, arte, ciencia y filosofia. Aun el trabajo del genio representa ideas, sentimientos e ideales comúnmente sostenidos en una determinada época y en un determinado entorno cultural [...] Es el medio en el que se realiza la comprensión de otras personas y de sus expresiones de vida". (W. Dilthey, "The 
Hermeneutas contemporáneos como H.G. Gadamer y Paul Ricoeur, siguiendo a Heidegger, han desarrollado esta tesis historicista, en el sentido de que no sólo el autor está condicionado por un contexto histórico y social específico, sino también el intérprete. ${ }^{15}$ Tales contextos histórico-sociales definen respecto al autor y al intérprete una correspondiente "situación" y "horizonte" hermenéuticos, que a su vez codeterminan el significado de la acción o texto que se interpreta. ${ }^{16}$ Consecuentemente, se renuncia a la idea de un significado original al cual deba de adecuarse la interpretación.

Situación y horizonte hermenéuticos son términos complementarios: "Situación" refiere a los "prejuicios" heredados por el intérprete (o autor) que subyacen en su contexto, mientras que "horizonte" alude a la direccionalidad y alcance de los "prejuicios" para proyectarse más allá de su situación inmediata. Así, para Gadamer los prejuicios no sólo son factores que limitan y condicionan la comprensión de las culturas ajenas o distantes, sino, sobre todo, condiciones que tienden un puente para su comprensión.

Para Gadamer los prejuicios vigentes en una determinada situación y horizonte hermenéuticos son resultado del desarrollo histórico de la "tradición", la cual constituye la fuente de legitimidad de los prejuicios:

Lo consagrado por la tradición y por el pasado posee una autoridad que se ha hecho anónima, y nuestro ser histórico y finito está determinado por el hecho de que la autoridad de lo transmitido, y no sólo lo que se acepta razonadamente tiene poder sobre nuestra acción y sobre nuestro comportamiento. ${ }^{17}$

Lejos de considerar la tradición contraria a la razón y a la libertad (como esta cita podría sugerir erróneamente), Gadamer afirma tajante que "La tradición siempre es también un momento de la libertad y de la historia" y que "la tradición es esencialmente conservación, y como tal nunca deja

Understanding of Other Persons and Their Life-Expressions" en Kurt Muller-Volmer (ed.), The Hermeneutics Reader, Continuum, Nueva York, 1988, p. 155.)

15 Sobre este punto véase el cap. 9 de Verdad y método, que considero el capítulo más importante del libro de Gadamer. En particular, el primer inciso de este capítulo denominado "El descubrimiento de la preestructura de la comprensión por Heidegger" es muy ilustrativo de este punto.

16 Cfr. P. Ricoeur "The Model of the Text. Meaningfull Action as a Text", en su Hermeneutics and the Human Sciences, Cambridge University Press, 1985. Sobre este mismo tópico Gadamer afirma: "El verdadero sentido de un texto tal como éste se presenta a su intérprete no depende del aspecto puramente ocasional que representa el autor y su público originario. $O$ por lo menos no se agota en esto. Pues este sentido está siempre determinado también por la situación histórica del intérprete, y en consecuencia por todo el proceso histórico." Gadamer, Verdad y método, Ediciones Sígueme, Salamanca, 1977, cap. 9, p. 366.

17 Gadamer, op. cit., p. 348. 
de estar presente la razón [...] tan libre como en la transformación y la innovación". ${ }^{18}$

Así pues, para Gadamer la comprensión se presenta como una "fusión de los horizontes" del autor y del intérprete en la que los prejuicios que guían la comprensión son puestos en cuestionamiento y pueden ser tanto conservados como suplantados. Esta decisión, aunque depende básicamente del intérprete, hay que verla "menos como una acción de la subjetividad que como un desplazarse uno mismo hacia un acontecer de la tradición". ${ }^{19}$

En este sentido, a través de la interpretación del pasado desde el horizonte hermenéutico del presente, la tradición misma se desarrolla progresivamente, cambiando en algo los prejuicios heredados y conformando así la nueva situación y horizonte hermenéuticos de los que partirán futuras interpretaciones. Este movimiento es lo que constituye para Gadamer el "círculo hermenéutico" a través del cual la tradición progresa:

El círculo no es, pues, de naturaleza formal; no es meramente subjetivo ni objetivo, sino que describe la comprensión como la interpretación del movimiento de la tradición y del movimiento del intérprete. La anticipación del sentido que guía nuestra comprensión de un texto no es un acto de la subjetividad, sino que se determina desde la comunidad que nos une con la tradición. ${ }^{20}$

Para Gadamer, las decisiones que se toman en el interior de una comunidad ligada a un determinado momento de la tradición están basadas en una racionalidad práctica más que teórica. Por ello, Gadamer considera que el saber moral de la "phrónesis" desarrollado por Aristóteles "se nos muestra como una especie de modelo de los problemas inherentes a la tarea hermenéutica". ${ }^{21}$ En este sentido, la racionalidad de la decisión de conservar o cambiar determinados prejuicios de la tradición ha de verse como un "buen juicio" surgido del diálogo y la comunicación dentro de la comunidad a la que pertenece el intérprete. "El buen juicio que involucra la phrónesis, no es un argumento demostrativo. Buen juicio se refiere aquí a un atributo juicioso del que juzga recta y equitativamente. El que posee buen juicio está dispuesto a reconocer el derecho de la situación concreta del otro... "22

18 Ibidem., p. 349-350.

19 Ibidem., p. 360 .

20 Ibidem., p. 363.

21 Ibidem., p. 396.

22 Ibidem., p. 396, obsérvense las analogías entre la noción de buen juicio aristotélico elaborado por Gadamer y la idea "de buen sentido" de Duhem. 


\section{Conclusiones}

Quisiera subrayar algunos de los puntos más importantes de convergencia entre la filosofía de la ciencia anglosajona pospositivista y la hermenéutica filosófica desarrollada a partir de Heidegger.

a) Para las dos tradiciones filosóficas los presupuestos de diverso orden (conceptuales, teóricos, ontológicos, metodológicos) juegan un papel determinante en el desarrollo de las teorías o de las interpretaciones.

b) Tanto las teorías científicas como las interpretaciones de la historia tienen que evaluarse en función de su contribución al progreso de la tradición a la que pertenecen.

c) Las tradiciones de investigación, en el campo de las ciencias naturales o socio-históricas, evolucionan paulatinamente combinándose tanto la conservación y tenacidad como el cambio y la innovación.

d) La decisión de mantener o cambiar determinados contenidos de la tradición no puede hacerse en función de reglas o criterios metodológicos precisos. Esta decisión se basa más bien en la comunicación y establecimiento de consensos en la comunidad que sustenta la tradición en cuestión. La racionalidad de este tipo de acuerdos responde más a una argumentación práctica, a un buen sentido, y no a metodologías que pretendan demostrar la verdad o falsedad de las hipótesis o de las interpretaciones.

Así pues, el desarrollo del buen juicio es el factor determinante para el progreso de las tradiciones. Las mejores oportunidades para desarrollar el buen sentido surgen cuando se dan encuentros entre tradiciones distintas, o entre voces distintas de una supuesta misma tradición, que cuestionan los presupuestos fundamentales de la tradición propia.

Estos puntos de convergencia o analogía entre las tradiciones hermenéuticas y pospositivista de la filosofía de la ciencia constituyen un buen indicio para explorar por caminos distintos una nueva noción general de la racionalidad científica, tanto en el ámbito de las ciencias naturales, como de las ciencias socio-históricas.

En términos más específicos, no se trata en realidad de elucidar un nuevo concepto de racionalidad, sino más bien de rescatar y revalorar la virtud de la prudencia, la sabiduría práctica aristotélica, la capacidad de juicio $^{23}$ como lugar y fundamento de la racionalidad científica, no sólo para

23 Analizando el concepto de juicio en Aristóteles, Kant, Arendt y Gadamer, Ronald Beiner lo define en términos que me parecen muy pertinentes en este trabajo: "El juicio es una capacidad natural de los seres humanos que, potencialmente, puede ser compartida por todos. Nos capacita para evaluar los detalles sin depender de las reglas ni de técnicos gobernados 
las ciencias sociales y las humanidades, como lo propone Gadamer, sino también para las ciencias naturales. Se abre así la posibilidad de elaborar una concepción general de la racionalidad cientifica, tomando como fundamento no la argumentación metódica y demostrativa, sino la argumentación comunicativa, pública, deliberativa, no demostrativa, pero sí convincente, que tradicionalmente se ha asociado al saber práctico y no a las teorías cientificas. 